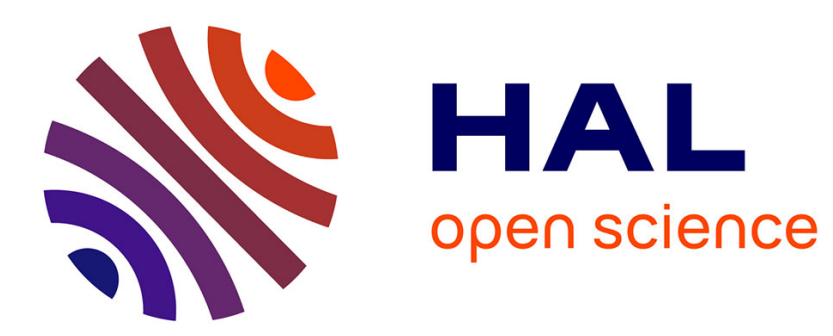

\title{
L'égalité dans les systèmes scolaires : effet école ou effet société?
}

Vincent Dupriez, Xavier Dumay

\section{To cite this version:}

Vincent Dupriez, Xavier Dumay. L'égalité dans les systèmes scolaires: effet école ou effet société?. 2004. halshs-00603497

\section{HAL Id: halshs-00603497 https://shs.hal.science/halshs-00603497}

Submitted on 25 Jun 2011

HAL is a multi-disciplinary open access archive for the deposit and dissemination of scientific research documents, whether they are published or not. The documents may come from teaching and research institutions in France or abroad, or from public or private research centers.
L'archive ouverte pluridisciplinaire HAL, est destinée au dépôt et à la diffusion de documents scientifiques de niveau recherche, publiés ou non, émanant des établissements d'enseignement et de recherche français ou étrangers, des laboratoires publics ou privés. 


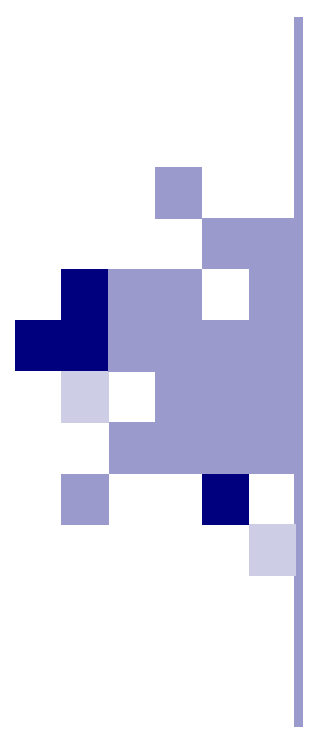

Les Cahiers de Recherche en Education et Formation

\section{L'ÉGALITÉ DANS LES SYSTÈMES SCOLAIRES : EFFET ÉCOLE OU EFFET SOCIÉTÉ ?}

Vincent Dupriez et Xavier Dumay

N³1 •OCOTBRE 2004• 
L'éducation et la formation constituent des enjeux fondamentaux pour la société contemporaine. Deux équipes de recherche à l'UCL se préoccupent de ces questions : le GIRSEF et la CPU.

Le GIRSEF est un groupe de recherche pluridisciplinaire fondé en 1998 afin d'étudier les sytèmes d'éducation et de formation. L'attention est portée notamment sur l'évaluation de leurs résultats en termes d'équité et d'efficacité, sur leurs modes de fonctionnement et de régulation, sur les politiques publiques à leur endroit, les logiques des acteurs principaux ou encore sur le fonctionnement local des organisations de formation ou l'engagement et la motivation des apprenants. Sur le plan empirique, ses recherches portent essentiellement sur le niveau primaire et secondaire d'enseignement, mais aussi sur l'enseignement supérieur et la formation d'adultes.

La Chaire de Pédagogie Universitaire (CPU) a été créée en mai 2001 et a reçu le label de Chaire UNESCO sur l'Enseignement Supérieur en septembre 2002. Elle assure également le secrétariat et la coordination du réseau européen des chaires Unesco sur l'Enseignement supérieur. Elle a pour mission de contribuer à la promotion de la qualité de la pédagogie universitaire à l'UCL, par le biais de la recherche dans le domaine et de l'enseignement (DES en pédagogie universitaire).

La série des Cahiers de recherche en Education et Formation était précédemment publiée sous le nom de "Cahiers de recherche du GIRSEF ». Cette série a pour objectif de diffuser les résultats des travaux menés au sein de la CPU et du GIRSEF auprès d'un public de chercheurs en sciences de l'éducation et de la formation ainsi qu'auprès des acteurs et décideurs de ces deux mondes.

L'ensemble de la série est téléchargeable gratuitement depuis les sites du GIRSEF(www.girsef.ucl.ac.be), de la CPU (www.cpu.psp.ucl.ac) ainsi qu' I6DOC (www.i6doc.com). 


\section{$C P U$}

\section{Table des matières}

1. INTRODUCTION

2. LES HYPOTHÈSES

3. LES DONNÉES

4. LES RÉSULTATS

4.1. Inégalités sociales et inégalités scolaires

4.2. Différenciation scolaire et inégalités

4.3. L'écart entre les inégalités scolaires à 10 ans et à 15 ans

5. DISCUSSION

6. CONCLUSION

RÉFÉRENCES BIBLIOGRAPHIQUES 
Les Cahiers de Recherche en Éducation et Formation - $n{ }^{\circ} 31-$ Octobre 2004

\section{Introduction}

Un des modes de travail de l'éducation comparée est d'étudier les structures scolaires de différents pays et d'essayer de rapporter les différences observées à des variations dans les performances scolaires des pays étudiés. De manière assez classique, cette comparaison de performances est étudiée en référence à des critères d'efficacité, d'efficience ou d'égalité, traités isolément ou de manière complémentaire. La multiplication des bases de données internationales de mesure des acquis des élèves tend évidemment à faciliter ce travail. Malgré les limites de ces outils standardisés de collecte d'informations, ils représentent en effet une ressource particulièrement intéressante pour documenter l'état des apprentissages dans différents systèmes scolaires et la manière dont ces apprentissages sont distribués parmi les élèves des pays considérés.

Plusieurs études préalables centrées sur la question de l'égalité dans les systèmes scolaires ont ainsi attiré l'attention sur le fait que les systèmes scolaires de type intégré sont globalement plus égalitaires que les systèmes scolaires de type différencié. Qu'entend-on par là ? Un système scolaire intégré se caractérise par une structure commune à tous les élèves (sans filières) sur une longue durée, un nombre de cours à options très limité au sein de cette structure commune et un recours faible ou inexistant au redoublement. II s'agit donc de systèmes scolaires qui, de manière structurelle, refusent le plus longtemps possible de séparer les élèves en fonction de leurs performances. Autrement dit, le principe est de laisser suffisamment de temps à l'école pour aller contre les différences de ressources culturelles des familles et d'apporter à chaque élève des chances réelles de réussite avant d'opérer toute forme de sélection. A l'opposé, on parle d'enseignement différencié pour évoquer des systèmes scolaires avec des filières organisées de manière précoce, un recours important aux orientations et aux options et un usage important du redoublement comme outil de gestion des parcours scolaires.
Aux deux extrémités de cet axe enseignement intégré versus enseignement différencié, il est assez aisé de placer d'une part les pays scandinaves, avec une structure unique pour tous les élèves jusque 16 ans et généralement une promotion automatique des élèves entre les années d'étude, et d'autre part l'Allemagne, l'Autriche, le Luxembourg, la Suisse et les Pays-Bas, avec un système de différenciation précoce des parcours des élèves. La plupart des systèmes scolaires des autres pays européens pourraient être positionnés dans un troisième groupe, au centre de cet axe. Ces systèmes scolaires se caractérisent soit par la coexistence de plusieurs structures parallèles (comme les comprehensive school et les grammar school en Angleterre, par exemple) soit par un recours intensif aux options et orientations dans une structure apparemment commune (comme le collège unique en France, par exemple).

Cette classification étant posée, nous pouvons maintenant analyser les résultats de plusieurs études comparatives récentes qui ont mobilisé ce critère comme axe central d'analyse des liens entre politiques scolaires et état des inégalités. Sur la base d'une mesure des performances en mathématiques (TIMMS ${ }^{1}$ 1995), Zachary, Dupriez et Vandenberghe (2002) concluent par exemple que les pays avec une structure unique et longue sont clairement plus équitables que les pays à différenciation précoce. L'équité est ici appréhendée sous forme de la mesure du lien entre un indice des ressources socioculturelles des familles et le score de l'élève. Cette conception de l'équité renvoie à ce que nous appellerons dans la suite de ce texte l'(in)égalité des chances (Benadusi, 2001) : il s'agit d'appréhender dans quelle mesure le score d'un élève est dépendant des ressources sociales et culturelles de son environnement familial. Sauf indication contraire, c'est d'ailleurs à cette définition de l'égalité que nous nous référons chaque fois que ce terme sera utilisé dans ce texte. A vrai dire, depuis les travaux de Bourdieu et Passeron (1970), on sait que cette relation entre capi-

\footnotetext{
2 Third International Mathematics and Science Study 1995.
} 
tal culturel et performances scolaires est présente, mais son degré d'intensité varie en fonction des pays, ce qui laisse entendre que l'organisation tant de la société que de l'école sont susceptibles d'affecter l'ampleur de la reproduction des inégalités.

Sur la base des données de PISA 2000 en compréhension à l'écrit, Duru-Bellat, Mons et Suchaut (2004) ont également été attentifs à l'intensité du lien entre la richesse matérielle et culturelle des familles et les scores des élèves. Pour chacun des pays étudiés, ils obtiennent un coefficient qui mesure l'ampleur de ce lien, autrement dit l'ampleur de l'inégalité des chances. Ils construisent par ailleurs une variable agrégée qui prend en compte le taux de redoublements en primaire, la brièveté du tronc commun et l'ampleur de la ségrégation scolaire entre établissements. II s'agit donc d'un indicateur du degré de différenciation au sein de chaque système scolaire. Ils constatent enfin que cet indicateur de différenciation est significativement corrélé $(r=0,63)$ à l'indicateur d'inégalité des chances. Ils soulignent dès lors dans leur conclusion que tout groupement par niveau ou par filières distinctes, mais aussi toute forme de ségrégation entre établissements, tend à accroître l'inégalité sociale de performances. Ils constatent par ailleurs que les pays « à culture de différenciation » n'améliorent pas pour autant le niveau moyen des scores des élèves. Au contraire, il existe une relation négative significative $(r=-0,33)$ entre cet indice de différenciation et le score moyen des pays en compréhension de l'écrit.

Crahay (2003) rend compte de résultats très semblables en s'appuyant sur différentes bases de données dont TIMMS 1995 et l'étude de compréhension à l'écrit (Reading Literacy - 1990), deux enquêtes internationales menées par l'IEA ${ }^{2}$. Fondamentalement, il en conclut que l'école n'a pas besoin de sélectionner pour être efficace et qu'au contraire les pays qui reportent le plus tard possible toute forme de sélection sont non seulement plus égalitaires, mais ils sont aussi parmi les plus efficaces de l'Union européenne. Il ajoute également qu'une éducation de lon-

\footnotetext{
${ }^{2}$ International association for the Evaluation of educational Achievement.
}

\section{$C P U$}

gue durée pour tous ne semble pas s'opposer à la formation d'élites, et que la meilleure façon de produire un grand nombre d'élèves brillants est de s'appuyer sur une structure scolaire intégrée. Gorard et Smith (2004), après une analyse des données de PISA 2000 concluent dans le même sens : "Nationally comprehensive systems of schools tend to produce narrower social differences in intake and outcomes. Systems with more differentiation lead to greater gaps in attainment between social groups. " (Gorard \& Smith, 2004, p. 25).

En fin de compte, ces diverses études tendent à montrer que les pays à différenciation précoce sont moins efficaces et plus inégalitaires que les autres, du moins si l'égalité est appréhendée en termes d'égalité des chances. Une critique qui peut être formulée à l'encontre de cette analyse, c'est que les systèmes scolaires apparaissant comme les plus égalitaires ne sont pas seulement des systèmes d'enseignement intégré, ce sont aussi des systèmes scolaires "nichés " dans des pays plus soucieux que d'autres de l'égalité entre les personnes. On ne peut en effet pas ignorer, quel que soit le mode d'opérationnalisation de la variable, que ce sont classiquement les pays scandinaves qui apparaissent comme le prototype d'une logique d'intégration. Dès lors, la question suivante mérite d'être posée : dans quelle mesure le caractère égalitaire de ces systèmes scolaires repose-t-il sur un effet école, attribuable en partie à la structure du système, ou plutôt sur un effet société, attribuable aux caractéristiques sociales et politiques de ces pays?

Dans une certaine mesure, cette question ne peut recevoir de réponse pleinement satisfaisante. II est d'ailleurs probable que l'ensemble de ces caractéristiques forme un système cohérent. Autrement dit, nous pourrions partir du postulat qu'une société a le système scolaire qu'elle mérite et que les politiques éducatives d'un pays représentent un indice parmi d'autres des valeurs qui sont portées dans chaque Etat. Malgré la difficulté inhérente à un tel exercice, l'objectif de ce texte est cependant d'assumer cette question et de se doter d'une méthode d'analyse visant à arbitrer entre l'effet école et l'effet société. 


\section{Les hypothèses}

Plusieurs études récentes font donc apparaître que les systèmes scolaires des pays scandinaves sont plus égalitaires que d'autres, dans le sens du moins où la réussite scolaire à l'âge de 14/15 ans est dans ces pays moins associée qu'ailleurs aux ressources socioculturelles des familles. Dans ce texte, nous mettons en concurrence deux hypothèses pour rendre compte de ce constat. La première considère qu'il s'agit fondamentalement d'un effet société et que le caractère égalitaire de ces systèmes scolaires s'explique principalement par le caractère égalitaire des sociétés dans lesquelles ils s'inscrivent. La seconde hypothèse considère que c'est bien, conformément à ce qui ressort de la littérature, un effet lié à la structure de l'école et en particulier à la position de ces pays sur l'axe enseignement intégré versus enseignement différencié.

Pour pouvoir arbitrer entre ces deux hypothèses, il s'agit de concevoir des modes de traitement des données où les déductions faites à partir de ces hypothèses s'éloignent et aboutissent si possible à des prédictions opposées. Nous proposons ci-dessous trois situations qui rencontrent cette exigence. Dans un premier temps, il s'agit de tester la relation entre un indice des inégalités de revenus dans le pays (indice de Gini) et l'état des inégalités scolaires à 15 ans. Ensuite, plutôt que d'isoler les pays scandinaves des autres sur l'axe enseignement intégré / enseignement différencié, nous proposons de considérer cette variable comme une variable continue et de mesure l'ampleur du lien entre cette variable et l'état des inégalités dans chacun des pays. Dans ce sens, nous serons particulièrement attentifs à observer ce qui se passe à l'autre extrémité de la variable, dans les pays à différenciation précoce. Enfin, nous terminerons en nous intéressant non pas à l'état des inégalités à l'école à 15 ans, mais à l'écart entre l'état des inégalités vers l'âge de 9/10 ans et celui observé à 15 ans. Si la structure scolaire produit effectivement un effet spécifique, on devrait observer dans les pays "intégrés " une réduction des inégalités entre ces deux mesures plus fortes qu'ailleurs. Nous précisons dans le tableau 1 les prédictions élaborées à partir des hypothèses initiales.

Tableau 1 : Effet école ou effet société ?

\begin{tabular}{|l|l|l|}
\hline & \multicolumn{1}{|c|}{ Effet société } & \multicolumn{1}{c|}{ Effet école } \\
\hline $\begin{array}{l}\text { Lien entre un indice d'inégalités } \\
\text { dans la société et un indice d'inéga- } \\
\text { lités à l'école à } 15 \text { ans }\end{array}$ & Corrélation forte entre les deux indices & $\begin{array}{l}\text { Absence de corrélation entre ces indi- } \\
\text { ces ou lien faible }\end{array}$ \\
\hline $\begin{array}{l}\text { Lien entre un indice de différencia- } \\
\text { tion scolaire et un indice d'inégali- } \\
\text { tés à l'école à } 15 \text { ans }\end{array}$ & $\begin{array}{l}\text { Absence de corrélation entre ces deux } \\
\text { indices }\end{array}$ & Corrélation forte entre ces deux indices \\
\hline $\begin{array}{l}\text { Mesure de l'écart entre l'état des } \\
\text { inégalités scolaires à } 9 \text { ans et à } 15 \\
\text { ans }\end{array}$ & $\begin{array}{l}\text { Cet écart est sans relation avec la struc- } \\
\text { ture du système scolaire }\end{array}$ & $\begin{array}{l}\text { Dans les pays à enseignement intégré, } \\
\text { la réduction des inégalités est plus forte } \\
\text { qu'ailleurs }\end{array}$ \\
\hline
\end{tabular}


Pour confronter ces hypothèses, nous nous appuyons sur un certain nombre d'analyses statistiques. Soulignons d'emblée que ces analyses ne rencontrent pas les critères des lois de la statistique inférentielle. Le nombre d'unités d'analyse (analyse 1 : $\mathrm{N}=21$; analyse $2: \mathrm{N}=16$; analyse $3: \mathrm{N}=11$ ) est à ce titre particulièrement critique et rend discutable toute généralisation à partir des relations entre variables observées ici. Dans une large mesure, ce n'est d'ailleurs pas notre objectif. Notre population de référence, comme nous le précisons ci-dessous, ce sont les systèmes scolaires des pays de l'Union euro-

\section{Les données}

Les résultats présentés dans cette recherche sont essentiellement issus d'analyses menées à partir de la base de données PISA produite en 2000 par I'OCDE. PISA - Program for International Student Assesment - est une enquête internationale menée à travers un large ensemble de pays industrialisés dont la plupart sont membres de l'OCDE. Elle a principalement pour objectif d'évaluer le niveau de compétences en lecture, en mathématiques et en sciences d'élèves de 15 ans au moyen de questionnaires standardisés. II est important de rappeler que l'âge des élèves est la référence d'échantillonnage dans PISA. Celui-ci représente en outre, dans plusieurs pays investigués, la fin de l'enseignement obligatoire pour les élèves à l'heure. Les scores de compétences sont standardisés sur une base internationale de telle sorte qu'ils aient une moyenne de 500 , et un écart-type de 100. En parallèle sont récoltées des données qui permettent de construire des "profils " élèves, où ceux-ci sont caractérisés par des variables socioéconomiques et socioculturelles.

Dans le but de comparer les résultats des élèves à $9 / 10$ ans avec leurs résultats à 15 ans (analyse 3 ), la base de données PIRLS développée en 2001 par I'IEA - Progression international reading literacy skills - a également été mobilisée. Cette enquête vise spécifiquement à évaluer le développement en péenne (UE) et notre base de données inclut de l'information sur presque la totalité de la population. Dans ce contexte, notre projet est d'observer au sein de la base de données l'intensité des liens entre variables (à travers un coefficient de corrélation le plus souvent), mais aussi de mener une analyse " qualitative » de données quantitatives, en donnant sens aux relations entre les variables investiguées à partir des positions relatives et contrastées des systèmes scolaires analysés. Ce n'est donc qu'à titre indicatif que, pour les principales analyses, nous indiquons le seuil de significativité des résultats. lecture d'enfants en quatrième année du cycle d'enseignement primaire. Contrairement à PISA donc, la référence d'échantillonnage est ici une année d'étude, et non l'âge des élèves. Les informations relatives à l'environnement social et culturel de l'élève sont obtenues dans PIRLS à travers un questionnaire complété par les parents de l'élève. Tout comme dans PISA, les scores sont standardisés sur base de l'échantillon international autour d'une moyenne de 500 et un écarttype de 100.

Seuls les scores de compétences en lecture ont été traités dans les analyses. Ce choix se justifie par un souci de cohérence lié au fait que PIRLS n'évalue que des résultats en lecture. Tous les systèmes scolaires européens représentés dans l'enquête PISA ont été pris en considération. Nous y avons également joint les données recueillies en Norvège, en Suisse, en Tchéquie et en Islande, pays européens qui ont un niveau de développement économique proche de celui des pays de l'UE, bien que cela soit moins évident dans le cas de la Tchéquie. Notre préoccupation en la matière était de veiller à ne pas comparer des réalités socio-économiques trop dissemblables et d'écarter de notre analyse des pays étudiés par PISA tels que le Brésil, la Russie ou la Corée, mais à ce point socialement et culturellement différent qu'il devient difficile d'isoler des facteurs explicatifs des différences à l'é- 
cole. De la sorte, nous réduisons évidemment les différences en termes de structure socio-économique et nous affaiblissons la puissance d'un éventuel effet société. La question de recherche que nous assumons est donc bien celle-ci : Au sein de pays au ni-

\section{Les résultats}

\subsection{Inégalités sociales et inégalités scolaires}

Les inégalités scolaires constatées à l'âge de 15 ans (à travers les données collectées par Pisa par exemple) peuvent a priori être imputées aux deux facteurs qui nous intéressent dans cette étude : un effet propre de la structure scolaire et/ou un effet des inégalités présentes dans la société. veau de richesse relativement proche (fondamentalement, les pays de l'UE), les inégalités scolaires sont-elles attribuables à un effet propre des systèmes scolaires, ou à un effet des inégalités sociales de la société dans laquelle ils s'inscrivent?
Dans cette première analyse, nous explorons l'effet des inégalités sociales sur les inégalités scolaires. Si l'hypothèse scolaire est correcte, on s'attend à ne trouver qu'un faible lien ou une absence de lien entre inégalités scolaires et inégalités sociales. Si l'hypothèse non-scolaire est correcte, on devrait observer un lien positif entre les deux paramètres étudiés.

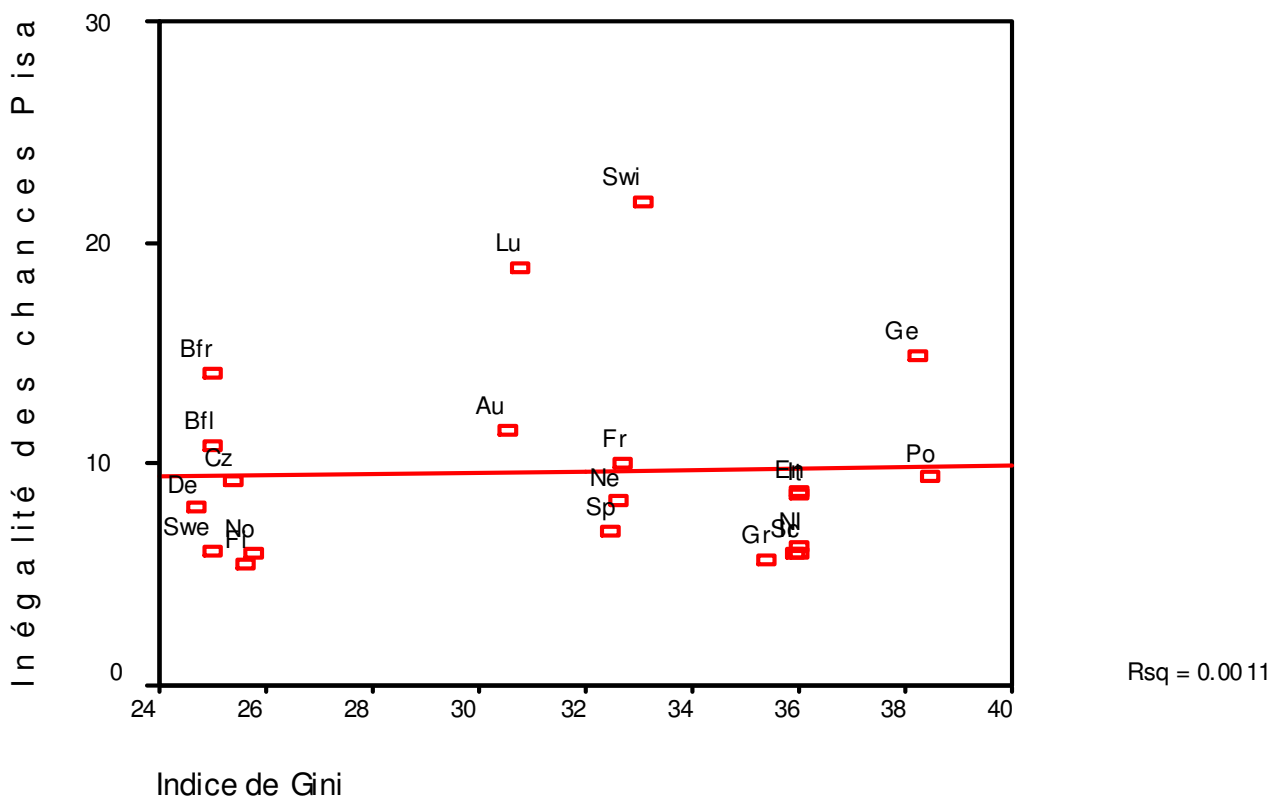

Figure 1 : Inégalités sociales et inégalités scolaires 
Pour réaliser cette analyse, nous avons utilisé l'indice de Gini ${ }^{3}$ comme indicateur des inégalités de revenu dans les pays considérés et nous avons construit un indicateur de l'inégalité des chances à l'école, à partir des données collectées par Pisa 2000 en compréhension à la lecture. Cet indice correspond au coefficient de détermination ( $\mathrm{R}^{2}$ ajusté) d'une équation où, pour chaque pays, le score des élèves est prédit à partir du diplôme de la mère (enseignement post-secondaire ou non), du langage parlé à domicile (langue de l'école ou non) et d'un indice des ressources économiques de la famille ${ }^{4}$. Plus élevé est ce coefficient de détermination, plus élevée est dans ce pays la relation entre ressources familiales et performances des élèves. Autrement dit, plus élevée est l'inégalité des chances.

Nous présentons sur la figure 1 la relation entre ces deux variables. De manière assez claire, il en ressort que cette relation est extrêmement faible. Rappelons que nous travaillons ici uniquement sur les pays européens et que, à l'échelle mondiale, ces pays se caractérisent par une faible inégalité de revenus et par une faible variance entre pays. II est probable que, à partir d'un échantillon de pays plus contrastés, on aurait obtenu une relation plus forte entre ces deux paramètres. Mais, à l'échelle de I'Union européenne en tout cas, il apparaît qu'une mesure de l'inégalité de revenus telle que l'indice de Gini n'est pas associée à l'état des inégalités scolaires. Ce constat se voit confirmé par une analyse similaire de cette relation menée à partir des données PIRLS ( $\mathrm{N}$ $=10 ; r=0.09 ;$ n.s.). L'état des inégalités à un moment antérieur du cursus scolaire ne semble donc pas davantage lié à l'équiréparition des revenus.

\footnotetext{
${ }^{3}$ L'indice de Gini est un indice d'équirépartition. Il évalue le niveau de concentration des biens (ici, les revenus de la population) en mesurant l'écart par rapport à une situation de répartition équitable. Par construction, il varie entre 1 et 100. Une valeur élevée révèle une distribution inégalitaire. Source des données pour cet indice : Rapport mondial sur le développement humain, PNUD, 2003.

${ }^{4}$ La variable Hisei dans Pisa : Highest international socioeconomic index.
}

\subsection{Différenciation scolaire et inégalités}

Dans cette analyse, nous testons la relation entre le caractère intégré ou différencié du système scolaire et l'état des inégalités. Mais, plutôt que d'isoler les pays scandinaves, comme prototypes d'un enseignement intégré, nous utilisons ici à l'instar de Duru-Bellat et al. (2004) un indicateur de différenciation qui permet d'attribuer une valeur numérique à chaque pays considéré. Cet indicateur est un score factoriel construit à partir de trois variables: l'âge de la première orientation dans chaque système scolaire, le pourcentage d'élèves en retard scolaire à 15 ans $^{5}$ et un indice de ségrégation académique entre établissements ${ }^{6}$, à 15 ans. Nous prenons donc en considération un indice de différenciation lié à la structure même du système scolaire et deux indices qui reflètent l'état de la différenciation réelle : le recours plus ou moins important au redoublement et les différences entre établissements au regard des acquis scolaires des élèves. Comme les analystes des systèmes scolaires le savent bien, ces trois paramètres sont extrêmement liés et révèlent la tendance de systèmes scolaires à adopter une logique de différenciation ou une logique d'intégration. Cette forte interaction entre les variables se traduit ici par la capacité du facteur de différenciation à « extraire » $74.3 \%$ de la variance présente sur les trois variables initiales.

Les résultats de l'analyse sont présentés sur la figure 2 : le lien entre le facteur de différenciation et l'état des inégalités à 15 ans est très élevé et statistiquement significatif $(r=0,84 ; p>0,001)$, malgré le fait que nous travaillons sur un nombre d'unités d'analyse très faible $(N=16)$. Ce résultat, associé à celui de la première analyse, plaide évidemment pour accorder du crédit à l'hypothèse scolaire. On se doit en tout cas

\footnotetext{
${ }^{5}$ En raison de l'absence d'infomations fiables concernant le taux de retard scolaire en Suisse et au Luxembourg, ces deux pays ont été retirés de l'analyse.

${ }^{6}$ Cet indice de dissimilarité entre écoles est proposé par Dupriez et Vandenberghe (2004) à partir des données de Pisa 2000. Il se définit comme la proportion d'une population $k$ donnée (ici, les élèves dont les résultats à l'épreuve PISA sont faibles, la mère peu éduquée, et le profil socioéconomique des parents faible) d'une zone géographique donnée qu'il y lieu de déplacer si l'on veut atteindre la situation d'égale répartition entre écoles.
} 
de constater qu'il existe une relation étroite entre le niveau de différenciation scolaire et l'ampleur des inégalités dans les systèmes scolaires. Et cette relation n'est pas seulement observée pour les pays scandinaves : on observe assez nettement sur la figure 2 une relation de type linéaire entre les deux variables.

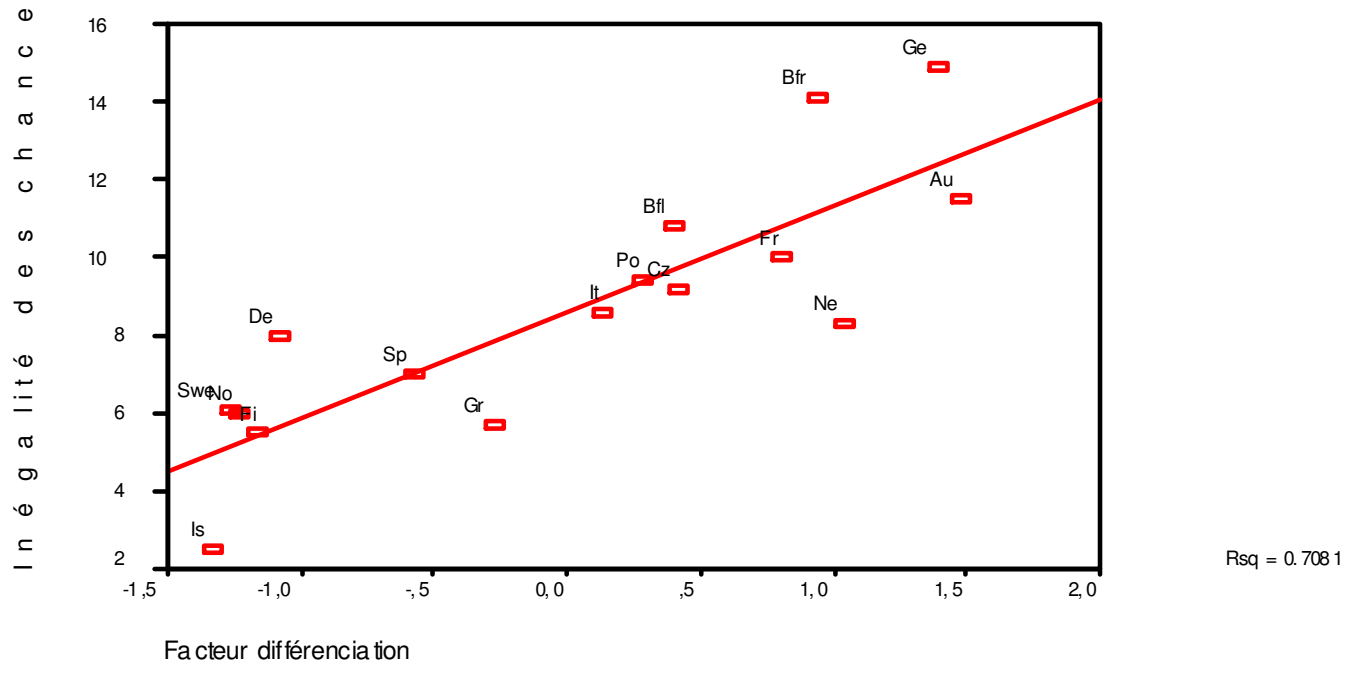

Figure 2 : Différenciation scolaire et inégalité des chances

On pourrait cependant opposer à une explication (trop) scolaire de ce constat que la corrélation n'a pas valeur de preuve et que le lien de causalité va peut-être dans le sens inverse de celui que suppose l'hypothèse scolaire. Autrement dit, ce serait l'homogénéité des résultats scolaires et les faibles inégalités à l'école qui permettent, dans certains pays, d'avoir une structure commune longue et de reporter le plus tard possible l'organisation de filières. Pour avancer par rapport à une telle objection, il devient indispensable de prendre en compte l'état des inégalités et de la distribution des résultats scolaires non pas à 15 ans, mais beaucoup plus tôt dans la scolarité, à un moment où tous les systèmes scolaires ont une structure commune pour tous les enfants C'est ce que nous proposons dans la troisième analyse.
4.3. L'écart entre les inégalités scolaires à 10 ans et à 15 ans

Cette dernière analyse va s'intéresser non pas à l'état des inégalités à l'âge de 15 ans, mais d'une part à l'état des inégalités plus tôt dans la scolarité et d'autre part à l'écart entre l'état des inégalités présentes dans l'enseignement primaire et la situation observée à 15 ans. Si la structure scolaire est bien un facteur déterminant de l'état des inégalités, on doit s'attendre non seulement à ce que à 15 ans, il y ait moins d'inégalité dans les pays intégrés (ce que nous avons montré dans la seconde analyse), mais aussi à observer dans ces derniers pays une réduction des inégalités entre l'école primaire et l'école secondaire plus forte qu'ailleurs. Dans l'hypothèse d'un effet société dominant, on devrait au contraire observer dans les pays égalitaires à 15 ans plus d'égalité dès l'enseignement primaire et dès lors une différence entre l'enseignement 
primaire et secondaire qui ne serait pas plus forte qu'ailleurs.

Pour développer une telle analyse, nous avons besoin de comparer des indices d'inégalité, construits à partir de deux prises d'informations distinctes : l'état des inégalités vers la fin de l'enseignement obligatoire (15 ans) et l'état des inégalités au début de l'enseignement primaire. Malheureusement, la plupart des études internationales telles que Pisa ne réalisent plus cette double collecte de données. Nous avons donc opté pour une comparaison entre des indices construits à partir de deux bases de données différentes : Pisa 2000 pour l'état des inégalités à 15 ans et PIRLS pour l'état des inégalités vers 9/10 ans. Une telle analyse ne peut bien entendu être menée qu'à partir des pays qui ont participé aux deux enquêtes internationales, ce qui réduit notre échantillon à un groupe de onze systèmes scolaires.

Vu la spécificité de cette hypothèse et l'intérêt pour une mesure de la dispersion des scores dans l'enseignement primaire, nous prenons ici en considération deux conceptions de l'inégalité : outre l'inégalité des chances, nous proposons un indice de l'inégalité de résultats. Celui-ci correspond au rapport, au sein de chaque pays, entre le premier et le neuvième décile de la distribution internationale.

Tableau 2 : Evolution des inégalités scolaires entre des mesures opérées sur des élèves de10 et de 15 ans

\begin{tabular}{|l|c|c|c|c|c|c|}
\hline & \multicolumn{3}{|c|}{ Inégalité de résultats } & \multicolumn{3}{c|}{ Inégalité des chances $^{\mathbf{7}}$} \\
\hline & 10 ans & 15 ans & Écart & 10 ans & 15 ans & Écart \\
\hline Allemagne (10) & 1.38 & 1.81 & +0.43 & 17,2 & 14,9 & $-2,3$ \\
Rép.Tchèque (11) & 1.34 & 1.64 & +0.30 & 14,1 & 9,2 & $-4,9$ \\
Pays-Bas (12) & 1.27 & 1.56 & +0.29 & 12,5 & 8,3 & $-4,2$ \\
& & & $\mathbf{+ 0 . 3 4}$ & & & $\mathbf{- 3 , 8}$ \\
\hline France (14) & 1.39 & 1.62 & +0.23 & 17,3 & 10 & $-7,3$ \\
Italie (14) & 1.38 & 1.62 & +0.24 & 6,4 & 8,6 & $+2,2$ \\
Grèce (15) & 1.40 & 1.71 & +0.31 & 13,5 & 5,7 & $-7,8$ \\
& & & $\mathbf{+ 0 . 2 6}$ & & & $\mathbf{4 , 3}$ \\
\hline Angleterre (16) & 1.49 & 1.64 & +0.15 & 11,7 & 8,7 & $-3,0$ \\
Ecosse (16) & 1.49 & 1.62 & +0.13 & 11,9 & 6,0 & $-5,9$ \\
Suède (16) & 1.32 & 1.61 & +0.29 & 9,1 & 6,1 & -3 \\
Norvège (16) & 1.52 & 1.69 & +0.17 & 13,3 & 6,0 & $-\mathbf{7 , 3}$ \\
Islande (16) & 1.44 & 1.60 & +0.16 & 13,2 & 2,5 & $-10,7$ \\
& & & $\mathbf{+ 0 . 1 8}$ & & & $\mathbf{- 5 , 9 8}$ \\
\hline
\end{tabular}

\footnotetext{
${ }^{7}$ Tout comme pour Pisa, l'égalité des chances dans PIRLS correspond à la valeur du coefficient de détermination d'une équation dont la variable dépendante est le score, et les prédicteurs le diplôme de la mère, la langue parlée à domicile et un indice de la richesse des familles. Ces données sont collectées, dans PIRLS, à partir d'un questionnaire adressé aux parents.
} 
Ces résultats sont extrêmement intéressants au regard de la problématique qui nous occupe. Si dans un premier temps, on s'intéresse à l'état des inégalités à 10 ans et à la présence éventuelle d'une structure commune de longue durée, on peut constater que les pays qui ont fait le choix politique d'un enseignement intégré et d'une structure unique de longue durée ( $1^{\text {ère }}$ orientation à 16 ans) ne sont pas les pays où il y aurait au départ le plus d'égalité à l'école. Au contraire, si l'on se réfère à l'égalité de résultats en particulier et donc à la dispersion des scores en quatrième année de l'école primaire, c'est dans les pays à orientation tardive qu'on observe les plus grandes différences entre élèves. Dans ce groupe de pays, la Suède est le seul système scolaire de notre échantillon où l'on observe, dès l'âge de 10 ans, une situation (relative) de faible dispersion des scores et de faible inégalité des chances. La situation observée dans ces pays, permet de manière assez convaincante de rejeter l'objection formulée à l'issue de notre seconde analyse.

Dans une posture critique face à l'hypothèse d'un effet école, nous évoquions en effet la possibilité que la relation forte entre les variables différenciation et inégalités scolaires pouvait également être interprétée comme une influence de la seconde variable sur la première. Autrement dit, la structure peu différenciée de ces systèmes scolaires pourrait être comprise comme une réponse à une situation initiale de faible inégalité de résultats et de faible inégalité des chances. Mais, les données présentées dans le tableau 2 mettent à mal cette interprétation : le caractère intégré ou différencié des systèmes scolaires ne s'explique pas, sur la base de nos données du moins, par l'état de l'égalité à l'école dans l'ensei- gnement primaire. Au contraire, l'Angleterre, l'Ecosse, la Norvège et dans une certaine mesure l'Islande connaissent au départ une inégalité de résultats plus importante que dans les autres pays étudiés et ont malgré tout mis en place une structure commune de longue durée.

Dans un second temps, c'est l'écart entre les deux mesures d'inégalité qui nous intéresse. Dans la perspective d'un effet école et plus précisément d'un effet de la structure du curriculum, nous avons posé que dans les pays à structure intégrée, les inégalités se réduisent entre 10 et 15 ans de manière plus forte qu'ailleurs. Si nous analysons la situation pays par pays, malgré l'une ou l'autre situation atypique, la tendance va bien dans le sens de cette hypothèse. La situation est sans doute encore plus claire lorsque, comme nous le faisons dans le tableau 2, nous regroupons les pays en fonction de l'âge de la première orientation scolaire : la différence est incontestable entre les deux groupes extrêmes. Quel que soit l'état initial des inégalités à l'école, les pays à orientation tardive (16 ans) évoluent de manière plus favorable que les pays à orientation précoce (à 10,11 ou 12 ans), au regard d'un objectif de réduction des inégalités. Fondamentalement, la tendance suggérée par ce tableau indique que si les pays à orientation tardive sont plus égalitaires que les autres, ce n'est pas parce qu'ils l'étaient déjà dès l'enseignement primaire, mais car leur mode de fonctionnement leur permet d'atténuer, davantage qu'ailleurs, les inégalités. A ce titre, la situation de l'Ecosse, de la Norvège et de l'Islande est particulièrement intéressante : ces pays connaissent une situation de forte inégalité à l'école primaire, adoptent une structure commune de longue durée et réduisent significativement l'état des inégalités. 


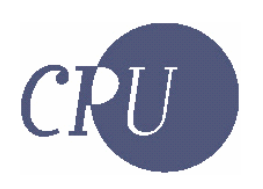

\section{Discussion}

Ces analyses successives tendent à montrer que l'état des inégalités scolaires à 15 ans semble attribuable, au moins en partie, à l'organisation d'un enseignement différencié, et ce de manière d'autant plus nette que sont comparés des pays fortement contrastés par rapport à cette dimension des politiques scolaires. Ce constat corrobore les résultats des investigations empiriques menées précédemment dans le champ de l'éducation comparée (Zachary \& Dupriez, 1999 ; Crahay, 2003 ; DuruBellat et collègues, 2004 ; Gorard \& Smith, 2004 ; Demeuse, Crahay \& Monseur, 2001).

Nos analyses permettent par ailleurs de faire apparaître de manière plus nette la spécificité de cet effet de la structure scolaire, indépendamment de l'état des inégalités dans la société, du moins pour l'ensemble des pays européens considérés dans cette base de données. Nous avons en effet montré l'absence de relation entre les inégalités à l'école et les inégalités dans la société appréhendées à partir de l'indice de Gini d'inégalités de revenus, tant pour des élèves de 15 ans que pour des élèves de 10 ans. Nous avons également montré que l'organisation d'un enseignement différencié a tendance à augmenter, ou du moins à ne pas diminuer, les inégalités scolaires de départ, alors qu'un enseignement de type intégré semble produire l'effet inverse.

Dans une certaine mesure, le résultat le plus étonnant de nos analyses, c'est l'absence de lien entre l'indice de concentration des revenus et l'inégalité des chances à l'école. Stricto sensu, ce résultat laisse entendre que l'ampleur de l'inégalité des chances à l'école n'est guère liée aux inégalités socioéconomiques dans la société. Bien entendu, ce résultat s'explique partiellement par les pays retenus dans cette étude. La variance entre pays européens par rapport à cet indice est faible au regard de ce qu'on observerait en incluant des pays d'autres continents. Cependant, des différences existent, mais elles ne s'accompagnent pas d'une variation linéaire sur notre indice d'inégalité des chances.
Pour approfondir cette analyse, il serait peut-être opportun de s'appuyer sur d'autres sources d'information, peut-être plus spécifiques. Par exemple, des indices d'accès aux soins de santé ou au logement mériteraient d'être croisés avec des indices d'égalité à l'école. Ils permettraient de mieux documenter l'interrogation que nous avons formulée et d'aider à répondre à une préoccupation fondamentale de politique publique (Maurin, 2002) : pour rendre l'école (et/ou la société) plus égalitaire, est-il préférable d'investir dans l'école ou plutôt dans d'autres secteurs affectant le bien-être de la population?

Il est également important de relever que cette étude est fondamentalement de type synchronique, même si nous introduisons deux mesures de l'égalité à l'école. II serait souhaitable, mais extrêmement complexe, de mener une étude diachronique qui prenne en compte l'évolution des inégalités à l'école, parallèlement à l'évolution des politiques éducatives et des transformations sociales, respectant dès lors davantage l'historicité des phénomènes étudiés. A ce jour, l'étude longitudinale la plus sérieuse sur une problématique semblable est celle de Shavit et Blosfeld (1993). Son objectif majeur était d'apprécier la capacité des 11 pays étudiés à atténuer les inégalités sociales dans les trajectoires scolaires et l'accès aux niveaux éducatifs les plus élevés. Et les auteurs constatent que la Suède est le seul pays à avoir clairement réduit de telles inégalités. S'interrogeant sur les raisons de ce succès suédois, Erikson et Jonsson (1996, cités par Benadusi 2001) considèrent que trois facteurs gouvernent les inégalités éducatives entre les classes sociales:

- les différences entre enfants de classes sociales différentes dans leurs compétences scolaires, dues à des différences dans les opportunités d'apprentissage hors école ;

- les différences dans la propension de ces élèves à rester à l'école jusqu'aux niveaux les plus élevés et dans les filières les plus prestigieuses;

- les effets de la démocratisation scolaire sur les taux globaux de scolarité. 
Et selon Erikson et Jonson toujours, c'est le second facteur qui permet de comprendre la performance suédoise dans une perspective comparative. Depuis 1946, la Suède évolue vers un enseignement intégré et depuis 1962, le système scolaire de ce pays est organisé sur base d'une structure unique de 7 à 16 ans pour tous les enfants du pays. Au-delà de l'impossibilité d'une orientation précoce vers des filières moins valorisées (et plus cette orientation est précoce, plus elle dépendra des ressources culturelles familiales), il faudrait également souligner l'impact de la structure unique sur les conditions d'apprentissage. Les recherches sur l'apprentissage en groupes homogènes ou hétérogènes (Dupriez \& Draelants, 2003 ; Ireson \& Hallam, 2003) ont en effet souligné que dans un groupe faible, les opportunités d'apprentissage en termes de temps d'apprentissage et de qualité de stimulation ne sont pas équivalentes à ce que l'on observe dans des groupes forts ou hétérogènes. En fin de compte, il est probable que le succès de l'enseignement intégré repose en bonne partie sur ces deux paramètres : l'impossibilité d'une orientation précoce trop dépendante de l'environnement familial d'une part et la qualité des conditions d'enseignement pour les plus faibles dans un contexte de groupes hétérogènes d'autre part.

Relevons enfin que, pour le groupe de pays que nous avons étudié, si un effet école semble bien présent, il est possible qu'il repose partiellement sur d'autres variables, non prises en compte dans cette étude. Autrement dit, la capacité d'égalisation des chances observées dans certains pays s'explique peut-être par d'autres paramètres scolaires que ceux que nous avons appréhendé. A ce titre, relevons tout d'abord que le facteur de différenciation construit pour la seconde analyse incorpore de facto une dimension parfois traitée séparément. En effet, une des variables introduites pour la construction du facteur est l'indice de dissimilarité entre écoles, calculé à partir du score des élèves en compréhension à l'écrit. Cet indice de dissimilarité révèle l'ampleur des différences entre écoles au regard de la proportion d'élèves au score faible qui y sont scolarisés. II révèle donc des différences empiriquement présentes. Mais, en termes de politiques éducatives, ces différences peuvent être rapportées à deux types de décision : la première porte sur la durée d'un tronc commun pour tous les élèves (dans la mesure où des élèves de filières différentes, là où elles existent, sont souvent scolarisés dans des établissements différents) et la seconde porte sur le mode de répartition des élèves entre écoles, indépendamment de la présence éventuelle de filières.

Si la première interrogation renvoie bien à la problématique traitée ici, la seconde interrogation est étroitement articulée à la question du libre choix de l'établissement et de l'impact de celui-ci à la fois sur la qualité de l'éducation (Vandenberghe, 1999), sur la ségrégation entre établissements (Gewirtz, Ball \& Bowe, $1995)$ et sur les inégalités à l'école. Mais d'autres paramètres pourraient également être appréhendés et étudiés comme possibles prédicteurs des inégalités scolaires. C'est dans une certaine mesure le difficile et ambitieux exercice réalisé par Duru-Bellat et al. (2004). Cet exercice est certainement utile et permet de mieux comprendre les constats opérés à partir de bases de données internationales. Par rapport à notre étude, la prise en compte d'autres facteurs (tels la décentralisation, les formes de supervision des enseignants, les conditions d'évaluation des élèves, ...) aiderait probablement à comprendre les variations entre pays semblables en termes d'intégration ou de différenciation, mais dont les résultats en termes d'égalité ne convergent pas. Mais un tel exercice dépasse l'objectif de ce papier, concentré sur le facteur probablement le plus puissant au regard de la question de l'égalité, et plus aisément objectivable et mesurable que d'autres paramètres des politiques éducatives. 


\section{Conclusion}

L'état des inégalités à l'école est-il le reflet des inégalités présentes dans la société ou dépend-il plutôt des caractéristiques propres des systèmes scolaires ? A travers trois analyses successives, nous avons tenté d'apporter une réponse à cette question. L'effet société était appréhendé à partir d'une mesure des inégalités de revenus dans les pays considérés. L'effet école était analysé à partir des positions respectives des pays sur l'axe enseignement intégré versus enseignement différencié. Et à chaque fois, notre réponse est favorable à l'hypothèse d'un effet école et défavorable à l'hypothèse d'un effet société.

S'agissant d'une étude corrélationnelle dans un domaine où les interactions entre de multiples paramètres sont complexes, nous devons bien entendu rester prudents. La convergence entre nos propres conclusions et les résultats de recherches antérieures nous pousse cependant à considérer que dans l'état actuel des connaissances sur les systèmes scolaires, on peut considérer qu'une logique de différenciation des élèves, telle qu'elle se manifeste dans des filières précoces, dans un usage intensif du redoublement et dans la ségrégation entre établissements est propice à un accroissement des inégalités à l'école. Au contraire, une logique d'intégration est associée à une diminution des inégalités.

Une telle conclusion pourrait pousser les décideurs et responsables éducatifs soucieux d'égalité à adopter et à importer dans leurs pays les paramètres qui semblent être décisifs dans le mode de fonctionnement de certains systèmes scolaires. Nous pensons qu'une telle orientation peut certainement représenter un objectif à long terme, mais qu'il faut rester prudent face à la capacité des acteurs scolaires à s'approprier un modèle venu d'ailleurs. Dans un certain sens, l'effet société est bien présent, même s'il n'apparaît pas dans la mesure que nous avons réalisée. L'effet société est sans doute, parmi des pays économiquement proches comme ceux de l'Union européenne, plus subtil : il passe par des représentations, des valeurs et des repères communs qui ren- dent peu ou prou crédibles la transformation des structures scolaires. A la lumière des néoinstitutionnalistes (Powell \& Di Maggio, 1991), on pourrait parler de dispositifs cognitifs et normatifs qui affectent la manière de penser et d'agir au sein du champ scolaire. Et dans ce sens, les caractéristiques de la structure scolaire sont révélatrices des valeurs d'une société et de la manière dont le rôle de l'école a été défini en son sein.

Se contenter d'importer une caractéristique structurelle (la durée du tronc commun, par exemple) est dès lors problématique, si un travail parallèle, mais autrement plus complexe, n'est pas réalisé pour travailler avec l'ensemble des acteurs scolaires la signification d'un tel changement et la transformation des pratiques socio-pédagogiques qui doit l'accompagner. Les évolutions récentes des politiques éducatives en Belgique francophone sont à ce titre assez illustratives. Dès 1994 et 1995, des initiatives sont prises pour prolonger un enseignement commun jusque 14 ans et implanter un principe de promotion automatique à l'intérieur de cycles d'étude pluriannuels. Mais, le pouvoir politique est déjà en train de déconstruire une telle réforme et a réintroduit la possibilité d'un redoublement à la fin de chaque année. L'analyse de Cornet et Dupriez (2004) suggère que cette marche arrière s'explique en bonne partie par l'absence d'un travail plus ample sur la signification politique de cette réforme et sur les conditions structurelles contradictoires de cette réforme : la tension forte entre un quasi-marché scolaire favorable à une logique de distinction et une réforme qui vise et qui prône l'égalité des résultats à la sortie de l'école.

Dans ce sens, oui, l'effet société existe bien, dans la mesure où c'est le contexte culturel et politique d'une société qui rend possible et donne sens à une structure scolaire spécifique. Mais l'effet scolaire existe aussi, dans la mesure où une fois la structure scolaire implantée et adoptée, les données empiriques font clairement apparaître que les structures intégrées sont plus égalitaires que les structures différenciées. 


\section{Références bibliographiques}

Benadusi L. (2001). Equity and education: a critical review of sociological research and thought. In W. Hutmacher, D. Cochrane, \& N. Bottani (Eds.), In pursuit of equity in education. (pp. 25-64). Dordrecht: Kluwer Academic Publishers.

Bourdieu, P., \& Passeron, J.-C. (1970). La Reproduction. Eléments pour une théorie du système d'enseignement. Paris: Editions de Minuit.

Cornet, J., \& Dupriez, V. (2004). Du prescit décrétal aux pratiques dans la classe: comment les enseignants perçoivent-ils et assument-ils les questions relatives à l'hétérogénéité des élèves ? In M. Frenay \& C. Maroy (Eds.), L'école, six ans après le décret "missions". (pp. 253-278). Louvain-la-Neuve: Presses universitaires de Louvain.

Crahay, M. (2003). Peut-on lutter contre l'échec scolaire? Bruxelles: De Boeck Université.

Demeuse, M., Crahay, M., \& Monseur, C. (2001). Efficiency and equity. In W. Hutmacher, D. Cochrane, \& N. Bottani (Eds.), In pursuit of equity in education. (pp. 65-92). Dordrecht: Kluwer Academic publishers.

Dupriez, V., \& Draelants, H. (2003). Classes homogènes versus classes hétérogènes: les apports de la recherche à l'analyse de la problématique. Les Cahiers de recherche du Girsef, $n^{\circ}$ 24, 3-22.

Duru-Bellat, M., Mons, N., \& Suchaut, B. (2004). Caractéristiques des systèmes éducatifs et compétences des jeunes à 15 ans. Les Cahiers de l'Iredu, 66, 1-158.

Erikson, R., \& Jonsson, J.O. (1996). Explaining class inequality in education: the swedish test case. In R. Erikson \& J.O. Jonsson (Eds.), Can education be equalized? Boulder: Westview Press.

Gewirtz, S., Ball, S., \& Bowe, R. (1995). Markets, choice and equity in education. Buckingham, Philadelphia: Open University Press.

Gorard, S., \& Smith, E. (2004). An international comparison of equity in education systems. Comparative Education, 40, 15-28.

Ireson, J., \& Hallan, S. (2001). Ability grouping in education. London: Paul Chapman Publishing.

Maurin, E. (2002). L'égalité des possibles. Paris: Seuil et la République des Idées.

Powell, V.W., \& Di Maggio, P.J. (1991). The new institutionalism in organizational analysis. Chicago and London: The university of Chicago Press.

Shavit, Y., \& Blossfeld, H.P. (1993). Persistent inequality. Changing educational attainment in thirteen countries. Boulder: Westview Press.

Vandenberghe, V. (1999). Combining market and bureaucratic control in education: an answer to market and bureaucratic failure ? Comparative Education, 35, 271-282. 


\section{CRU}

Zachary, M.D., Dupriez, V., \& Vandenberghe, V. (2002). Comparaison internationale de l'efficacité et de l'équité des systèmes d'enseignement. In D. de la Croix, F. Docquier, C. Mainguet, S. Perelman, \& E. Wasner (Eds.), Capital humain et dualisme sur le marché du travail. (pp. 259-279). Bruxelles: De Boeck.

Zachary, M.-D., \& Dupriez, V. (1999). Structures scolaires et équité pédagogique. In B. Gazier, J.L. Vutin, \& F. Audier (Eds.), L'économie sociale : formes d'organisation et institutions. Paris: L'Harmattan 
Annexe $\mathbf{n}^{\circ} \mathbf{1}$ : Les données

\begin{tabular}{|c|c|c|c|c|c|c|}
\hline & $\begin{array}{l}\text { Indice de } \\
\text { Gini }\end{array}$ & $\begin{array}{c}\text { Inégalité des } \\
\text { chances } \\
\text { (PISA) }\end{array}$ & $\begin{array}{c}\text { Inégalité des } \\
\text { chances } \\
\text { (PIRLS) }\end{array}$ & $\begin{array}{c}\text { Inégalité des } \\
\text { résultats } \\
\text { (PISA) }\end{array}$ & $\begin{array}{c}\text { Inégalité des ré- } \\
\text { sultats } \\
\text { (PIRLS) }\end{array}$ & $\begin{array}{c}\text { Facteur différen- } \\
\text { ciation }^{8}\end{array}$ \\
\hline Allemagne (GE) & 38.2 & 14.9 & 10.2 & 1.81 & 1.38 & 1.39 \\
\hline Angleterre (EN) & 36 & 8.7 & 11.7 & 1.64 & 1.49 & \\
\hline Autriche (AU) & 30.5 & 11.5 & & 1.60 & & 1.48 \\
\hline $\begin{array}{l}\text { Belgique francophone } \\
\text { (BFR) }\end{array}$ & 25 & 14.1 & & 1.88 & & 0.94 \\
\hline $\begin{array}{l}\text { Belgique neérlando- } \\
\text { phone (BFL) }\end{array}$ & 25 & 10.8 & & 1.61 & & 0.41 \\
\hline Danemark (DE) & 24.7 & 8 & & 1.65 & & -1.08 \\
\hline Ecosse (SC) & 36 & 6 & 11.9 & 1.62 & 1.49 & \\
\hline Espagne (SP) & 32.5 & 7 & & 1.57 & & -0.58 \\
\hline Finlande (FI) & 25.6 & 5.5 & & 1.52 & & -1.16 \\
\hline France (FR) & 32.7 & 10 & 17.3 & 1.62 & 1.39 & 0.81 \\
\hline Grèce (GR) & 35.4 & 5.7 & 13.5 & 1.71 & 1.40 & -0.27 \\
\hline Irlande (IR) & 36 & 6 & & 1.61 & & \\
\hline Irlande du Nord (NI) & 35.9 & 6.3 & & 1.70 & & \\
\hline Islande (IS) & & 2.5 & 13.2 & 1.60 & 1.44 & -1.33 \\
\hline Italie (IT) & 36 & 8.6 & 6.4 & 1.62 & 1.38 & 0.13 \\
\hline Luxembourg (Lu) & 30.8 & 18.9 & & 1.87 & & \\
\hline Norvège (NO) & 25.8 & 6 & 13.3 & 1.69 & 1.52 & -1.23 \\
\hline Portugal (PO) & 38.5 & 9.4 & & 1.71 & & 0.29 \\
\hline Pays-Bas (NE) & 32.6 & 8.3 & 12.5 & 1.56 & 1.27 & 1.04 \\
\hline Tchéquie (CZ) & 25.4 & 9.2 & 14.1 & 1.64 & 1.34 & 0.41 \\
\hline Suède (SWE) & 25 & 6.1 & 9.1 & 1.61 & 1.32 & -1.26 \\
\hline Suisse (SWI) & 33.1 & 21.9 & & 1.73 & & \\
\hline
\end{tabular}

${ }^{8}$ Pour rappel, ce facteur est construit sur base de trois variables : l'âge de la première orientation, un indice de dissimilarité entre établissements construit à partir de la base de données PISA et le pourcentage d'élèves en retard scolaire à 15 ans. Cette dernière variable a également été construite à partir de la base de données PISA. Cependant, les spécificités du calendrier scolaire ou des différences régionales au sein d'un pays ont parfois rendu incertaine la construction d'un taux de retard scolaire à partir d'une telle base de données. Pour trois pays, notre source d'information est externe à PISA. Il s'agit de la République Tchèque, de l'Allemagne et des Pays-Bas. Pour le Royaume-Uni, l'Irlande, la Suisse et le Luxembourg, nous n'avons pas pu obtenir d'informations fiables relatives à ce paramètre. 


\title{
Cahiers de Recherche du GIRSEF
}

\author{
Déjà Parus :
}

Thiéry F., Zachary M-D., De Villé Ph., Vandenberghe V. (1999) Enseignement initial : les enjeux du développement de l'enseignement supérieur sur fond de crise de l'enseignement secondaire qualifiant, Les cahiers de Recherche du GIRSEF, $n^{\circ} 1$.

Conter B., Maroy C., Urger F. (1999) Le développement de la formation professionnelle continue en Belgique francophone, Les cahiers de Recherche du GIRSEF, $\mathrm{n}^{\circ} 2$.

Vandenberghe V. (1999) Regard rétrospectif sur la dynamique des dépenses d'enseignement en Communauté française de Belgique :1988-1998, Les cahiers de Recherche du GIRSEF, $\mathrm{n}^{\circ} 3$.

Dupriez V., Maroy C. (1999) Politiques scolaires et coordination de l'action, Les cahiers de Recherche du GIRSEF, $n^{\circ} 4$.

Doray, P. (2000) Les articulations entre formation professionnelle initiale et formation continue au Québec :quelques pistes de travail, Les cahiers de Recherche du GIRSEF, $n^{\circ} 5$.

Casalfiore S. (2000) L'activité des enseignants en classe. Contribution à la compréhension de la réalité professionnelle des enseignants, Les cahiers de Recherche du GIRSEF, $\mathrm{n}^{\circ} 6$.

Vandenberghe V. et Zachary M-D. (2000) Efficacité-équité dans l'enseignement secondaire de la Communauté Wallonie Bruxelles : essai d'évaluation dans le cadre d'une comparaison internationale, Les cahiers de Recherche du GIRSEF, $\mathrm{n}{ }^{\circ}$.

Vandenberghe V. (2000) Enseignement et iniquité : singularités de la question en Communauté Wallonie-Bruxelles, Les cahiers de Recherche du GIRSEF, n8.

Guyot J.-L., Bonami M. (2000) Modes de structuration du travail professoral et logiques disciplinaires à l'Université, Les cahiers de Recherche du GIRSEF, $\mathrm{n}^{\circ} 9$.

Cattonar B. (2001) Les identités professionnelles enseignantes. Ebauche d'un cadre d'analyse. Les cahiers de Recherche du GIRSEF, $\mathrm{n}^{\circ} 10$.

Maroy C. et Doray, P. (2001) La construction des relations écoles / entreprises. Le cas de la formation en alternance en Communauté Française de Belgique et au Québec, Les cahiers de Recherche du GIRSEF, n¹1.

Maroy C. (2001) Le modèle du praticien réflexif à l'épreuve de l'enquête, Les cahiers de Recherche du GIRSEF, $\mathrm{n}{ }^{\circ} 12$.

Mangez E. (2002) Régulation et complexité des rapports familles - écoles, Les cahiers de Recherche du GIRSEF, ${ }^{\circ} 13$.

Waltenberg F. D. (2002) Polarisation et appariements sélectifs des individus. Etat de la question, Les cahiers de Recherche du GIRSEF, $\mathrm{n}^{\circ} 14$.

Si vous souhaitez obtenir d'autres numéros de ces cahiers, vous pouvez les obtenir au secrétariat du GIRSEF au prix unitaire de $6 €$, Place Montesquieu 1 bte 14, B-1348 Louvain-la-Neuve, Tél : 32-10-472066, Fax : 32-10472400, email : girsef@anso.ucl.ac.be

Un résumé des textes parus dans ces cahiers est disponible sur le Web à l'adresse suivante : http://www.girsef. ucl.ac.be/cahiers.html. 


\section{Cahiers de Recherche du GIRSEF (suite)}

Waltenberg F. D. et Vandenberghe V. (2002) Etat des lieux de mobilité des élèves en cours de scolarité primaire en Communauté française Wallonie - Bruxelles. Une analyse économique et quantitative, Les cahiers de Recherche du GIRSEF, $\mathrm{n}^{\circ} 15$.

Casalfiore S. (2002) Les petits conflits quotidiens dans les classes de l'enseignement secondaire. I. Nature et sens des transgressions sociales à l'origine des conflits dans la dyade enseignant-élève, Les cahiers de Recherche du GIRSEF, $\mathrm{n}{ }^{\circ} 16$

De Villé Ph. (2002) Equal opportunity in the educational system and the ethics of responsibilty, Les cahiers de Recherche du GIRSEF, $\mathrm{n}^{\circ 17}$

Maroy M. et Cattonar B. (2002) Professionnalisation ou déprofessionnalisation des enseignants ? Le cas de la Communauté française de Belgique. Les cahiers de Recherche du GIRSEF, n`18.

Dauphin N. et Verhoeven M. (2002) La mobilité scolaire au cœur des transformations du système scolaire. Les cahiers de Recherche du GIRSEF, n¹9.

Casalfiore S. (2003) Les petits conflits quotidiens dans les classes de l'enseignement secondaire. II. Nature des stratégies de résolution, Les cahiers de Recherche du GIRSEF, n²0

Casalfiore S. (2003) Les petits conflits quotidiens dans les classes de l'enseignement secondaire. III. Conception de l'autorité chez les élèves, Les cahiers de Recherche du GIRSEF, n²1

Vandenberghe V. (2003) Un enseignement secondaire technique et professionnel (dé)valorisé ?, Les cahiers de Recherche du GIRSEF, $\mathrm{n}{ }^{\circ 22}$

Dupriez V. (2003) La coordination du travail dans les établissements scolaires: les différentes voies de construction de l'accord, Les cahiers de Recherche du GIRSEF, $\mathrm{n}{ }^{\circ} 23$

Dupriez V. et Draelants H. (2003) Classes homogènes versus classes hétérogènes : les apports de la recherche à l'analyse de la problématique, Les cahiers de Recherche du GIRSEF, $\mathrm{n}^{\circ} 24$

Letor C. et Vandenberghe V. (2003) L'accès aux compétences est-il plus (ini)équitable que l'accès aux savoirs traditionnels ?, Les cahiers de Recherche du GIRSEF, $\mathrm{n}{ }^{\circ} 25$

Galand B. et Gillet M.-P. (2004) Le rôle du comportement de la direction dans l'engagement professionnel des enseignants, Les cahiers de Recherche du GIRSEF, n`26

Dupriez V. et Vandenberghe V. (2004) L'école en Communauté française de Belgique : de quelle inégalité parlons-nous? Les cahiers de Recherche du GIRSEF, $\mathrm{n}{ }^{\circ 27}$

Vandenberghe V. (2004) Achievement Effectiveness and Equity. The role of Tracking, Grade-Repetition and Inter-school Segregation, Les cahiers de Recherche du GIRSEF, $\mathrm{n}{ }^{\circ 28}$

Galand B. et Vanlede M. (2004) Le sentiment d'efficacité personnelle dans l'apprentissage et la formation : Quel rôle joue-t-il? D'où vient-il? Comment intervenir? Les cahiers de Recherche du GIRSEF, n²9

Vandenberghe V. (2004) Les tendances longues de l'accumulation du capital humain en Belgique, Les cahiers de Recherche du GIRSEF, $\mathrm{n}{ }^{\circ} 30$ 